\title{
MPI-Based Parallelized Model Order Reduction Algorithm
}

\author{
Igor Balk $^{1}$ and Sergei Zorin ${ }^{2}$ \\ ${ }^{1}$ R3 Logic Inc, 80 Sherman Street, Camridge MA 02140, USA \\ ibalkar3logic.com \\ ${ }^{2}$ Parametric Corp., Boston, MA, USA \\ szorin@ptc.com
}

\begin{abstract}
Progress in integrated circuits and packaging design has made significant changes in the requirements for modeling tools. In this paper described algorithm of parallelization of Krylov subspace model order reduction technique for "full-wave" electromagnetic simulation.
\end{abstract}

\section{Introduction}

The topic of model order reduction appears in many disciplines. In control systems, perhaps the most well known approaches are Truncated Balanced realizations and Hankel singular values. Other popular approaches are based on the modal properties of the system, such as the method of Selective Modal Analysis, which is widely used in power systems applications. Unfortunately, most of these methods require $\mathrm{O}\left(\mathrm{n}^{3}\right)$, where $\mathrm{n}$ is the number of unknowns, operations to generate reduced model since they require explicit knowledge of the entire eigenspectrum of the system as in selective modal analysis or the hankel singular values as in truncated balanced realization. In complicated packaging and interconnect problems for which $\mathrm{n}$ can be quite large, these methods would require days and gigabytes of memory to compute.

In the area of circuit simulation, asymptotic waveform evaluation has popularized the use of model order reduction. A robust approach for deriving moment matching reduced order models is the Arnoldi process. The Arnoldi process has its origin in eigenvalues computation but was used recently to generate moment matching reduced order models.

In this paper we will give a brief overview of Arnoldi-based passive algorithm for reduction of 'full-wave' electromagnetic models $[2,3]$ and then introduce MPI-based parallelization strategy.

\section{Overview of Krylov Subspace Model Order Reduction}

Lets consider a single input - single output linear system, where $A$ is the system matrix

$$
\begin{aligned}
& A \frac{d x}{d t}=x+b u \\
& y=c^{t} x
\end{aligned}
$$

$A$ here is $m \times m$ matrix and $b, c \in R^{m}$. 
From (1.1) the relation for the transfer function $H(s)$ in Laplace space can be written as

$$
H(s)=-c^{t}(I-s A)^{-1} b
$$

Transfer function $\mathrm{H}(\mathrm{s})$ can be expanded in a Taylor series around zero frequency,

$$
H(s)=\sum_{i=0}^{\infty} m_{i} s^{i}=\sum_{i=0}^{\infty}-c^{t} A^{-(i+1)} b s^{i}
$$

where $\mathrm{m}_{\mathrm{i}}$, the coefficient of the $i$-th term in the Taylor series, is known as the $i$-th moment of the transfer function. Most commonly used model order reduction algorithms utilize the Arnoldi method of fast generation of orthogonal basis for Krylov subspace defined as

$$
K_{q}(A, b)=\operatorname{span}\left\{b, A b, A^{2} b \ldots A^{q-1} b\right\}
$$

The general idea of this method is to generate $k$-th order orthogonalized Krylov subspace from the $(k-1)$-th order orthogonalized Krylov subspace. The last vector of $(k-1)$-th order from the orthogonalized subspace is multiplied by $A$ and then the resulting vector is orthogonalized with respect to the rest of the basis. After $n$ steps this algorithm returns a set of $n$ orthogonal vectors and we can construct a matrix $V_{n} \in R^{m \times n}$ and an $n \times n$ upper Hessenberg matrix $H_{n}$ such as

$$
A V_{n}=V_{n} H_{n}+h_{n+1, n} v_{n+1} e_{n}^{t}
$$

It's easy see that for $k<n$

$$
A^{k} b=\|b\|_{2} A^{k} V_{n} e_{1}=\|b\|_{2} V_{n} H_{n}^{k} e_{1}
$$

With this relation we can write that moments can be related to $H_{n}$ by

$$
m_{k}=c^{t} A^{k} b=\|b\|_{2} c^{t} V_{n} H_{n}^{k} e_{1}=c_{n}^{t} A_{n}^{k} b_{n}
$$

and the $\mathrm{n}$-th order Arnoldi based approximation to $\mathrm{H}(\mathrm{s})$ can be written as

$$
H(s) \approx\|b\| c^{t} V_{n}\left(I-s H_{n}\right)^{-1} e_{1}
$$

corresponding to the steady state representation $A_{n}=H_{n}, b_{n}=e_{1}$ and $c_{n}=\|b\| V_{n} c^{t}$.

And substitution to the representation of original system we will result in following equation:

And it is easy to see that

$$
\begin{aligned}
& V_{n}^{t} A V_{n} \frac{d x}{d t}=V_{n}^{t} x+V_{n}^{t} b u \\
& \tilde{y}=c^{t} V_{n}^{t} x
\end{aligned}
$$

$$
y \approx \tilde{y}
$$




\section{Guarantee Passive Algorithm for Full Wave Model}

Now lets assume that $\mathrm{A}$ is frequency dependent and that the transfer function is positive definite for all $\mathrm{s}$ with non-negative real part; i.e. $\operatorname{Re}(i, H(s) i) \varepsilon$ for some $\varepsilon>0$. This kind of problem is quite common in modeling of interconnect circuits or packaging devices at high frequencies when we have to use the 'full-wave' kernel to generate integral equation formulation. The standard Arnoldi based model order reduction algorithm, such as PRIMA, are unable to handle this type of problem, and has been a lot of work done in this field. We will introduce here a passive algorithm for Arnoldi based reduction of such systems.

In order to generate a reduced order model A(s) will be presented in a from:

$$
A(s)=\frac{1}{s^{p}+1} A_{t}+\frac{s^{p}}{s^{p}+1} A_{t}
$$

where $A_{t}$ is the power series expansion of $\mathrm{A}(\mathrm{s})$ with infinite radii of convergence. And first order infinite dimension matrix will be generated in a way similar to the formulation described in [1].

$$
\begin{gathered}
{\left[I+\frac{s}{s^{p}+1}\left|\begin{array}{cccc}
\tilde{A}_{0} & \tilde{A}_{1} & \cdots & \tilde{A}_{N} \\
-I & 0 & \cdots & 0 \\
\vdots & \ddots & \ddots & \vdots \\
0 & 0 & -I & 0
\end{array}\right|\left|\begin{array}{c}
x_{0} \\
x_{1} \\
\vdots \\
x_{N}
\end{array}\right|=\left|\begin{array}{c}
b \\
0 \\
\vdots \\
0
\end{array}\right|\right.} \\
y=\left[\begin{array}{llll}
c^{t} & 0 & \cdots & 0
\end{array}\right]\left|\begin{array}{c}
x_{0} \\
x_{1} \\
\vdots \\
x_{N}
\end{array}\right|
\end{gathered}
$$

where $x_{k}=s^{k} x$ and $\tilde{A}_{k}=A_{0}^{-1} A_{k+1}$ for $k+1<p$ and $\tilde{A}_{k}=A_{0}^{-1}\left(A_{k+1}+A_{k+1-p}\right)$ for $k+1 p$.

Arnoldi process can be used for the new systems with $\tilde{s}=\frac{s}{s^{p}+1}$. And model order reduction algorithm can be formalized as follows:

1. Calculate $A_{0}$

2. Set $n=0$ and set some $p>1$

3. Calculate zero order transfer function

4. Increase $n$ by 1

5. Calculate $A_{n}$

6. Update p such as $\left|\tilde{s} \max _{i, j} \tilde{A}_{i, j}\right|<1$ for any $A_{n}$ and $\tilde{s}$ 
7. Update $A_{l}$ for all $l$ from 0 to $n$

8. Calculate $n$-th order transfer function

9. Check convergence of transfer function

10. If converge calculate reduced model else back to step 4

Using the properties of power series expansion with infinite radii of convergence and the definition of passivity, it's possible to show that the introduced algorithm will produce a passive reduced model. Proof is based on the fact that we do not have to truncate the expansion of $A(s)$ in order to calculate the $n$-th order of the reduced model and that expansion of transfer function will converge for any value of $\tilde{S}$.

\section{Parallelization Strategy}

Is it can clearly can be seen from the matrix structure in Eq. (2.2) the problem has intuitive and easy to observe block structure. First and the easiest way of parallelization would be assigning each block to the separate processor and using one processor to reconstruct the reduced matrix. The disadvantage of this strategy is that it requires explicit computation of submatrixes $A_{i, j}$ and vector matrix products and is quite costly in terms of memory and processor time. The alternative approach does not require explicit computation of all $A_{i, j}$ utilizes the fact that right hand side has only $n$ none zero entries. Each submatrix $A_{i, j}$ is divided in to block and each block is assigned to the processor. Each processor performs computations within it's own block to calculate matrix vector product and then while receiving data from neighbors it computes boundary data for the processors with adjusted blocks. Thus we will get optimal performance as we are doing computations at the same time as waiting for new data.

\section{Computational Results and Conclusions}

Above-mentioned parallelization was implemented using MPI standard libraries for easy portability. Parallel performance results can be seen on Fig. 1. The test was performed on a cluster of Sun Sparc processors with shared memory. We used transmition line simulation in a frequency range from $1 \mathrm{MHz}$ to $7 \mathrm{GHz}$ for the test runs.

The results indicate that electromagnetic and signal integrity analysis can be performed faster and it makes simulation of large and complex 3D structures possible. The approach described in this paper requires minimal changes to the serial algorithm. We are currently are working on developing new more effective parallelization strategies. 


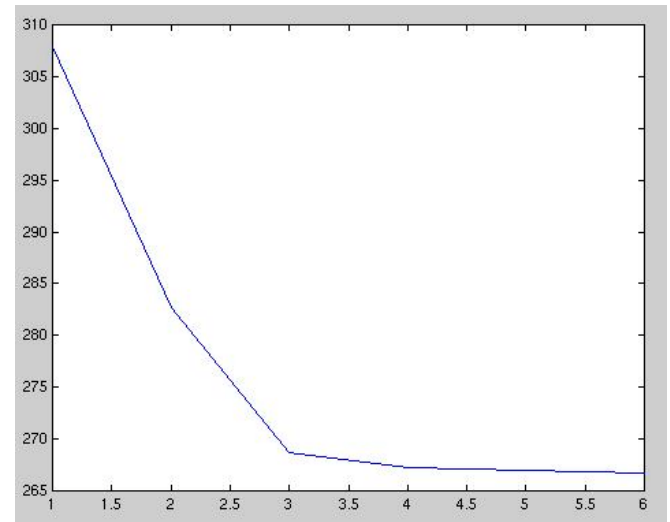

Fig. 1. Computational time vs. number of processors

\section{References}

1. Joel R. Phillips, Eli Chiprout and David D. Ling.: "Efficient full-wave electromagnetic analysis via model order reduction of fast integral transforms". 33rd DAC, Las Vegas 1996

2. Igor Balk: "Arnoldy based passive model order reduction algorithm". EPEP 2000, Scottsdale, 2000

3. Igor Balk, Yie He: "Full Wave Electromagnetic Analysis and Model Order Reduction for Complex Three Dimensional Structures”. MSM 2001, Hilton Head Island, 2001 